\title{
Braquiterapia com Cobalto60 para o tratamento do melanoma da úvea: análise dos fatores prognósticos para melhor resposta local
}

\author{
Cobalt - 60 brachytherapy for uveal melanoma: analysis of prognostic factors for local \\ response
}

\author{
Martha Motono Chojniak ${ }^{1}$ \\ Clelia Maria Erwenne ${ }^{2}$
}

\footnotetext{
${ }^{1}$ Mestre em oftalmologia pela Universidade Federal de São Paulo-EPM, Diretora do Departamento de Oftalmologia do Hospital do Câncer-A.C.Camargo.

${ }^{2}$ Doutora em Oftalmologia pela Universidade Federal de São Paulo-EPM, Chefe do serviço de tumores do Departamento de Oftalmologia do Hospital São Paulo.

Endereço para correspondência: Rua São Benedito, 984 - casa 5 - São Paulo (SP) CEP 04735-002.

E-mail: Chojniak@uol.com.br

Recebido para publicação em 19.02.2001

Aceito para publicação em 17.07.2001
}

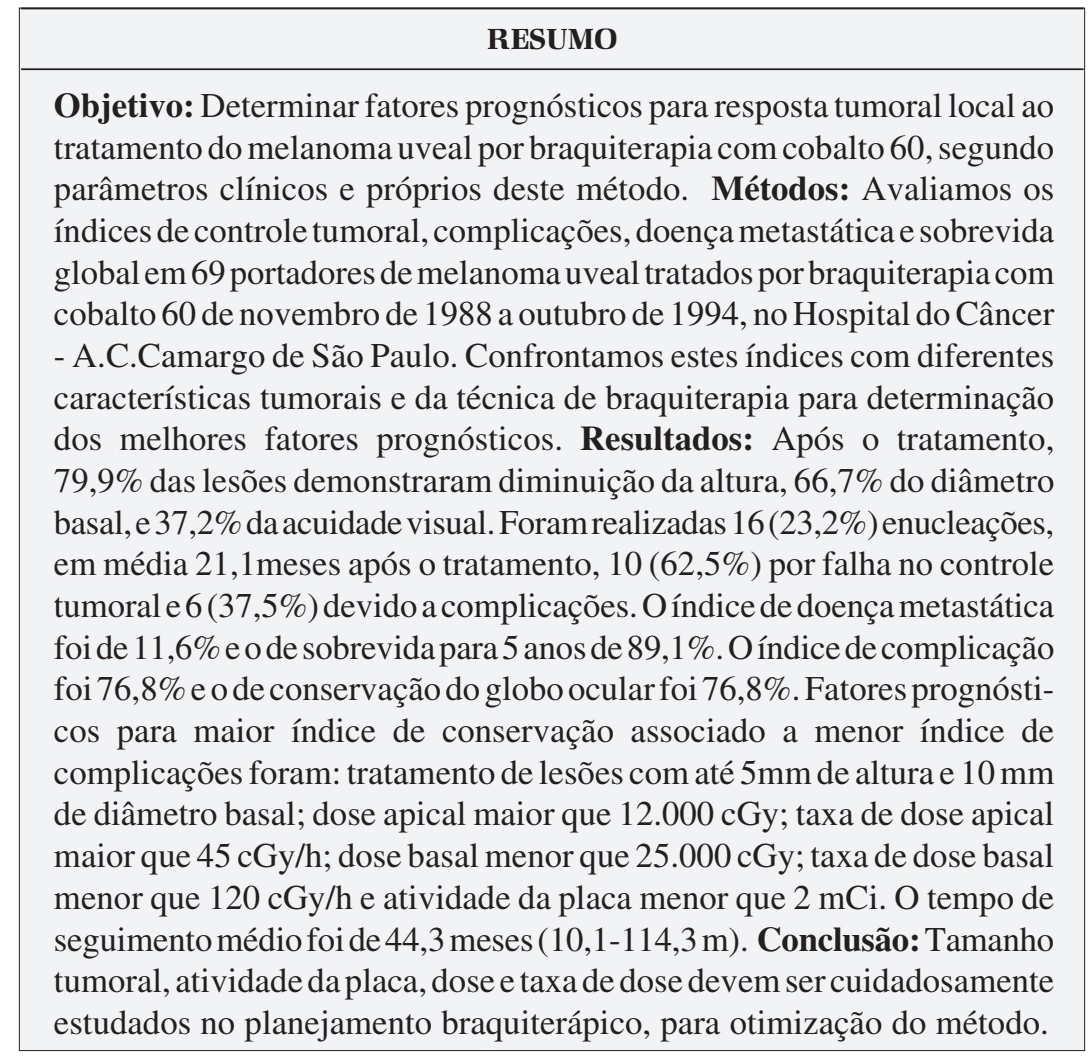

Descritores: Melanoma/quimioterapia; Neoplasiais uveais/quimioterapia; Cobalto/uso terapêutico; Prognóstico

\section{INTRODUÇÃO}

O melanoma da úvea é o tumor intra-ocular primário mais freqüente, constituindo $80 \%$ dos tumores oculares ${ }^{(1)}$. A incidência estimada é de seis novos casos/milhão/ano ${ }^{(2-3)}$. Acomete preferencialmente individuos acima dos 50 anos de idade e da raça branca ${ }^{(3)}$. Pode acometer qualquer porção da úvea, $97 \%$ apresentam-se na coróide posterior e/ou corpo ciliar, sendo os demais irianos $^{(3)}$. Apresentam-se clinicamente como espessamentos circuncritos da coróide, com formato "cupuliforme" ou "em cogumelo"(4), freqüentemente pigmentados. A doença metastática é a causa do óbito observada em aproximadamente $30 \%$ dos pacientes. A via de disseminação é a hematogênica e o fígado é o órgão mais freqüentemente acometido, seguido pelo pulmão ${ }^{(3,5)}$. 
A melhor terapêutica para o tratamento do melanoma da úvea ainda não está estabelecida. Diferentes opções podem ser utilizadas, sendo o principal fator determinante da escolha o tamanho da lesão tumoral.

A braquiterapia tem sido a principal terapêutica utilizada em muitos centros oncológicos no tratamento de melanomas médios e selecionados melanomas grandes. Representa um tipo de tratamento com radiação ionizante onde a fonte radioativa está em contato direto com o orgão a ser tratado; os aplicadores oftálmicos apresentam-se na forma de placas episclerais.

A literatura demostra não existir diferença significativa entre a taxa de sobrevida de pacientes tratados por enucleação ou por braquiterapia ${ }^{(6-7)}$; este motivo associado ao fato de possibilitar a conservação do globo ocular, muitas vezes com visão útil, levou a uma crescente preferência desta ${ }^{(8)}$.

Vários isótopos podem ser utilizados ${ }^{(9)}$ : Cobalto 60 , Rutenium $106^{(10)}$, Palladium $103^{(11)}$, Iridium $192^{(12)}$, Sumarium 145 e o Iodo $125^{(13)}$, considerado na atualidade o isótopo de eleição, tendo em vista a proteção radiológica que o mesmo proporciona para as estruturas anexas do globo ocular e para a equipe cirúrgica.

O Cobalto 60 é um emissor gama, com meia vida longa $(5,26$ anos) e boa penetração tecidual. Sua alta energia $(1,17$ - 1,33 $\mathrm{MeV}$ ) torna difícil o bloqueio de sua radiação para as estruturas oculares normais e para a equipe cirúrgica ${ }^{(9)}$. Por ser parte integrante do aplicador ocular, possibilita a reutilização da mesma placa inúmeras vezes, diminuindo os custos do tratamento $^{(9)}$.

As características braquiterápicas ideais para o tratamento do melanoma da úvea ainda não foram determinadas. A dose utilizada continua sendo semelhante a primeira sugerida em 1966: 8.000 a 10.000cGy no ápice da lesão e de 30.000 a 50.000 cGy na base, porém, o assunto é controverso ${ }^{(14-15)}$.

A literatura sobre o tratamento braquiterápico demonstra resultados visuais satisfatórios em $30 \%$ a $75 \%$ dos $\operatorname{casos}^{(12,16-18)}$. O índice de complicações relatado está em torno de $40 \%$, sendo as tardias as principais, ocorrendo frequentemente 2 anos após o tratamento ${ }^{(3)}$. O controle tumoral pode ser considerado bom, com falha relatada entre $4 \%$ e $33 \%$ (14-15,19-24). $^{2}$.

Fatores aparentemente relacionados com a falha do controle tumoral local e a ocorrência de complicações são: o tamanho ${ }^{(16,25)}$ e localização do tumor tratado $^{(15,22-23)}$, assim como a dose e taxa de dose radioativa utilizada ${ }^{(12,15,22)}$.

De forma geral, a expectativa de controle tumoral local para 5 anos é de $59 \%$ a $82 \%{ }^{(26)}$, o índice de sucesso no tratamento de $58,8 \%$ a $87,9 \%{ }^{(21,26)}$ e as taxas de sobrevida entre $80 \%$ e $88 \%$ para um seguimento de 5 anos e $66 \%-79 \%$ para $10 \operatorname{anos}^{(12,15,22)}$.

\section{OBJETIVO}

Determinação de fatores prognósticos para melhor resposta local em termos de conservação do globo ocular e complicações, no tratamento do melanoma da úvea por braquiterapia com Cobalto 60, segundo parâmetros clínicos e próprios do método braquiterápico.

\section{MÉTODO}

O material deste estudo foi obtido a partir de portadores de melanoma da úvea, tratados por braquiterapia com implante episcleral de Cobalto 60, no período de novembro de 1988 a outubro de 1994, no Hospital do Câncer - A.C.Camargo de São Paulo, tendo sido aprovado pela comissão de pesquisa e ética médica desta instituição.

Foram colhidos dados de prontuário e realizada reavaliação oftalmológica e sistêmica prospectiva.

Os registros incluíam: idade; sexo; raça; sintomas e duração; além de exame oftalmológico, onde foi inferido o diagnóstico de melanoma da úvea, que incluiu: medida da acuidade visual, biomicroscopia anterior em lâmpada de fenda, tonometria de aplanação, oftalmoscopia binocular indireta, ultrassonografia ocular $(10 \mathrm{mHz})$ e angiofluoresceinografia.

A braquiterapia utilizou placas radioativas episclerais de Cobalto 60 de procedência inglesa - Amersham International e objetivou uma dose radioativa de 10.000 cGy no ápice da lesão tumoral e 30.000 a 40.000cGy na base.

A programação braquiterápica foi realizada em conjunto ao serviço de física do Departamento de Radioterapia do Hospital do Câncer. Ao final da programação eram conhecidas as seguintes características radioterápicas em relação ao tratamento: Atividade da placa (mci), taxa de dose radioativa emitida por hora ao ápice do tumor (cGy/h), dose radioativa total no ápice (cGy), taxa de dose radioativa emitida por hora a base do tumor $(\mathrm{cGy} / \mathrm{h})$, dose radioativa total na base (cGy) e tempo de tratamento (horas).

Os procedimentos cirúrgicos de colocação e retirada das placas foram sempre realizados em sala cirúrgica especial, com proteção radiológica, segundo normas do CNEN (Conselho Nacional de Energia Nuclear), sob anestesia geral.

$O$ ato cirúrgico consistiu de: oftalmoscopia binocular indireta para localizar a lesão, transiluminação via transpupilar para evidenciar as margens tumorais, demarcação destas margens no tecido episcleral com caneta cirúrgica, posicionamento da placa em correto alinhamento com o tumor e sutura da placa no tecido escleral por meio do anodamento de pontos posicionados nas alças da placa. A resposta ao tratamento foi avaliada através da mesma propedêutica oftalmológica utilizada previamente ao tratamento, realizada no $30^{\circ}$ pós-operatório e trimestralmente a partir desta data.

Para a avaliação da resposta terapêutica local considerouse como controle tumoral local, definido pela estabilização ou diminuição das dimensões da lesão ao ultra-som com conservação do globo ocular.

A indicação de enucleação após o tratamento braquiterápico, ocorreu em duas situações clínicas: falha no controle tumoral local e presença de complicações que culminassem em olho cego e doloroso. 
Após a realização da enucleação, todo o espécime foi submetido a estudo anátomo-patológico macroscópico e microscópico.

A avaliação sistêmica para pesquisa de metástase foi realizada ao diagnóstico e semestralmente a partir desta data e incluiu: avaliação sérica de enzimas hepáticas, ultra-sonografia do abdômen superior e Raios-X de tórax frente e perfil.

Por ocasião da última avaliação os pacientes foram classificados segundo seu estado clínico em: vivo sem doença metastática, vivo com doença metastática, óbito pelo tumor e paciente perdido no seguimento (não comparecimento ao retorno por um período duas vezes maior do que o solicitado pelo médico, sem confirmação do óbito).

O estudo estatístico utilizou o software "STATA" (Stata Corp. 1995. Statistical Software: Release 4.0, College Station, Texas).

Foi realizada a estatística descritiva das diferentes variáveis clínicas e próprias do método terapêutico.

Para a determinação dos fatores prognósticos de melhor resposta local analisamos a associação dos índices de complicações e conservação do globo ocular em relação aos diferentes agrupamentos das variáveis clínicas (altura e base) e próprias do método terapêutico (dose e taxa de dose no ápice e base, atividade e tempo de tratamento), por meio de testes de hipóteses de Fisher e qui-quadrado com 95\% de significância.

Foi considerado como "melhor resposta local" a associação do maior índice de conservação do globo ocular ao menor índice de complicações.

A evolução sistêmica foi analisada pelo estado clínico na última avaliação e curvas atuariais de Kaplan-Meier para a sobrevida e doença metastática.

\section{RESULTADOS}

Este estudo avaliou um total de 69 pacientes portadores de melanoma maligno da úvea, seguidos a partir da data do tratamento braquiterápico por um tempo médio de 41,7 meses $(8,9$ 86,3 meses; mediana 38,6 meses).

Eram 41 pacientes $(59,4 \%)$ do sexo feminino e $28(40,6 \%)$ do masculino; com idade média de 50,4 anos (20-82 anos; mediana 50 anos). A raça branca representou $92,8 \%(n=64)$ da amostra, a parda $5,8 \%(n=4)$ e a oriental $1,4 \%(n=1)$.

Os sintomas referidos foram: baixa da acuidade visual $(47,5 \%)$, perda do campo visual $(15,2 \%)$, fotopsias $(12,1 \%)$, metamorfopsias $(6,1 \%)$, moscas volantes $(5 \%)$, hiperemia (1\%) e dor ocular (1\%). Em 3\% dos casos as queixas foram inespecíficas e em $9,1 \%$ a lesão tumoral foi encontrada como um achado de exame, em portadores assintomáticos. A duração da apresentação dos sintomas variou de 7 dias a 7 anos, com média de 9 meses (mediana 3 meses).

Eram pigmentados $97,1 \%(\mathrm{n}=67)$ das lesões e amelanóticos $2,9 \%(\mathrm{n}=2)$. As medidas das lesões tumorais por ocasião do diagnóstico mostraram altura média de 5,60mm $(1,50-13 \mathrm{~mm}$; mediana $5,40 \mathrm{~mm}$ ); o maior diâmetro basal com média de 10,84mm (5-16mm; mediana $11 \mathrm{~mm})$ e o menor diâmetro basal com média de 9,78mm (5-16 mm; mediana 9,90mm).

A acuidade visual do olho acometido por ocasião do diagnóstico foi pior que 20/200 em 13 pacientes $(18,8 \%)$ e após tratamento em 29 pacientes $(56 \%)$.

Após o tratamento ocorreu diminuição da altura em 79,7\% dos casos $(n=55)$, aumento em 18,8\% ( $n=13)$ e ausência de alteração desta medida em 1,5\% $(\mathrm{n}=1)$; tendo sido encontrado em média uma diminuição de 1,42 $\mathrm{mm}$ (mediana de 1,50mm).

O diâmetro basal máximo apresentou diminuição em 66,7\% dos casos $(n=46)$ e aumento de 20,3\% ( $n=14)$, não tendo sido possível a realização desta medida em $13 \%$ dos casos $(n=9)$, devido a pouca espessura da lesão, o que impossibilitou a individualização das margens tumorais pelo método ultra-sonográfico. A média de diminuição encontrada foi de $1,47 \mathrm{~mm}$ (mediana de 1,60mm).

A dose radioativa total no ápice teve média de $11.421 \mathrm{cGy}$ (3.630-21.692 cGy; mediana $10.320 \mathrm{cGy}$ ) e a taxa de dose radioativa/hora no ápice teve média de 52.432 cGy/h (16.020-105.815 cGy/h; mediana $50.679 \mathrm{cGy} / \mathrm{h}$ ).

A dose radioativa total na base teve média de $28.084 \mathrm{cGy}$ (13.780-47.147cGy; mediana $28.409 \mathrm{cGy})$ e a taxa de dose radioativa/hora na base teve média de 120.897 cGy/h (79.833$192.000 \mathrm{cGy} / \mathrm{h}$; mediana $117.491 \mathrm{cGy} / \mathrm{h})$.

A atividade das placas teve média de $2,03 \mathrm{mCi}(0,95-3,27$ $\mathrm{mCi}$ ) e o tempo de tratamento foi em média 243 horas (116-481 horas; mediana 216 horas).

Tanto o índice de conservação do globo ocular como o de complicações foram de 76,8\% (53 pacientes).

A frequiência das diferentes complicações foi: retinopatia da radiação $(56,5 \%)$, papilopatia da radiação $(34,8 \%)$, catarata radioinduzida $(26,1 \%)$, edema macular cistóide $(9 \%)$, hemorragia vítrea $(20,3 \%)$, glaucoma (13\%), oclusão venosa (13\%), descolamento da retina $(10,1 \%)$ e afinamento escleral $(2,9 \%)$. A tabela 1 mostra o intervalo de tempo decorrido entre o tratamento e o aparecimento das diferentes complicações.

Foram realizadas 16 enucleações $(23,2 \%)$ pós braquiterapia, $10(62,5 \%)$ por falha no controle tumoral local e $6(37,5 \%)$ por complicações que ocorreram, em média, 21,1 meses após o tratamento (2-50 meses; mediana de 12,6 meses). O estudo histopatológico confirmou o diagnóstico clínico de melanoma maligno da coróide em todos os casos.

A falha de controle tumoral local foi de $14,5 \%$.

Como estado clínico na "última revisão" tivemos: 60 pacientes $(87 \%)$ vivos e sem a presença de doença metastática, 3 $(4,3 \%)$ vivos e com a presença de doença metastática, 5 (7,2\%) tinham ido a óbito devido à presença da doença tumoral e 1 $(1,5 \%)$ foi perdido no seguimento.

A doença metastática ocorreu em 8 pacientes $(11,6 \%)$, em média 31,8 meses após o tratamento (2,9-65,3 meses; mediana 28,3 meses). O índice de metástase encontrado para 5 anos foi de $16,8 \%$ e para 7 anos, $39,3 \%$ (Figura 1 ).

$\mathrm{O}$ óbito devido à doença metastática ocorreu em 5 pacientes (7,3\%), em média 41,7 meses após o tratamento (15,5-72 meses; 


\begin{tabular}{|c|c|c|c|c|c|}
\hline $\begin{array}{l}\text { Complicação } \\
\mathrm{N}\end{array}$ & № Complicação & $\begin{array}{c}\text { Índice \% } \\
\text { Complicação }\end{array}$ & $\begin{array}{l}\text { Média tempo de } \\
\text { aparecimento meses } \\
\text { (Desvio padrão) }\end{array}$ & $\begin{array}{l}\text { Mediana tempo de } \\
\text { aparecimento meses }\end{array}$ & $\begin{array}{l}\text { Variação tempo de } \\
\text { aparecimento meses }\end{array}$ \\
\hline RT RD & 39 & $56,5 \%$ & $25,56(14,92)$ & 21 & $7-58$ \\
\hline PP RD & 24 & $34,8 \%$ & $31,83(15,61)$ & 33 & $6-59$ \\
\hline CATARATA & 18 & $26,1 \%$ & $28(19,61)$ & 21 & $5-72$ \\
\hline EMC & 18 & $26,1 \%$ & $30,27(19,77)$ & 31 & $6-72$ \\
\hline HEM VT & 14 & $20,3 \%$ & $26(14,13)$ & 22 & $1-48$ \\
\hline GLAUCOMA & 09 & $13 \%$ & $23(14,56)$ & 22 & $2-48$ \\
\hline OCL VEN & 09 & $13 \%$ & $27,22(15,05)$ & 21 & $10-52$ \\
\hline DR & 07 & $10,1 \%$ & $17,57(14,04)$ & 14 & $1-40$ \\
\hline AFIN ESCL & 02 & $2,9 \%$ & $7(7,07)$ & 7 & $2-12$ \\
\hline
\end{tabular}

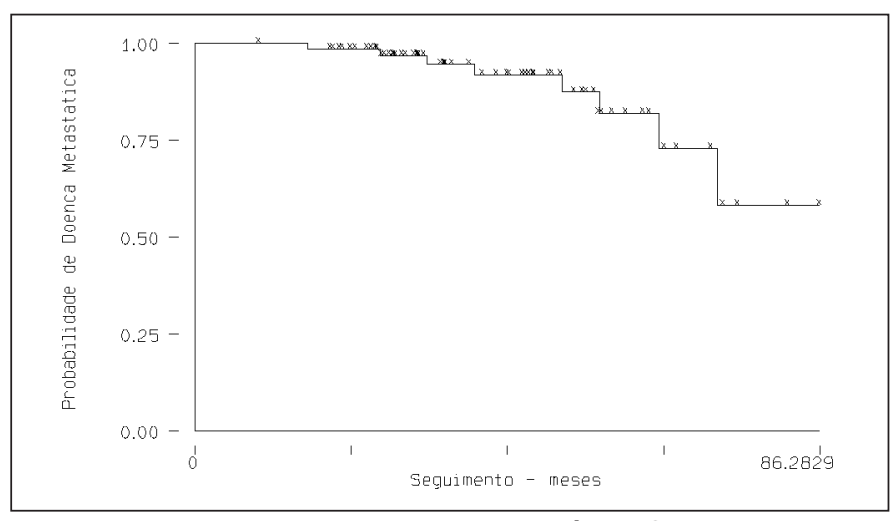

Figura 1 - Probabilidade de doença metastática. Curva atuarial

mediana 38,6 meses). O índice de sobrevida para 5 anos foi de $89,1 \%$ e para 7 anos, $71,3 \%$ (Figura 2).

$\mathrm{O}$ estudo estatístico mostrou que quanto maior foi a altura da lesão tratada, menor foi o índice de conservação $(\mathrm{P}=0,007)$ e maior o de complicações $(\mathrm{P}=0,031)$ (tabelas 2 e 3$)$. Observamos resultados semelhantes em relação ao diâmetro basal da lesão tumoral, onde quanto maior foi a base da lesão tratada, menor foi o índice de conservação $(\mathrm{P}=0,011)$ e maior o de complicações ( $\mathrm{P}=0,376)$, sendo este último sem significância estatística (tabelas 2 e 3 ).

O estudo estatístico mostrou que as lesões que receberam dose radioativa no ápice $>12.000 \mathrm{cGy}$ e taxa de dose radioativa/ hora no ápice $>45 \mathrm{cGy} / \mathrm{h}$, tiveram maior índice de conservação $(\mathrm{P}=0,033$ e $\mathrm{P}=0,029)$ e menor índice de complicações $(\mathrm{P}=0,008 \mathrm{e}$ $\mathrm{P}=0,057$ ) respectivamente (tabelas 2 e 3 ).

Os estudos estatísticos mostraram também que as lesões que receberam dose radioativa na base $\leq 25.000 \mathrm{cGy}$ e taxa de dose radioativa/hora na base $\leq 120 \mathrm{~Gy} / \mathrm{h}$, apresentaram o maior índice de conservação ( $\mathrm{P}=0,359$ e $\mathrm{P}=0,006)$, e menor índice de complicações $(\mathrm{P}=0,481 \mathrm{e} \mathrm{P}=0,016)$ respectivamente (tabelas $2 \mathrm{e} 3$ ).

As lesões tratadas por placas que apresentavam atividade $\leq 2 \mathrm{mCi}$ apresentaram maior índice de conservação, $(\mathrm{P}=0,002) \mathrm{e}$ menor índice de complicações $(\mathrm{P}=0,008)$ (tabelas 2 e 3 ).

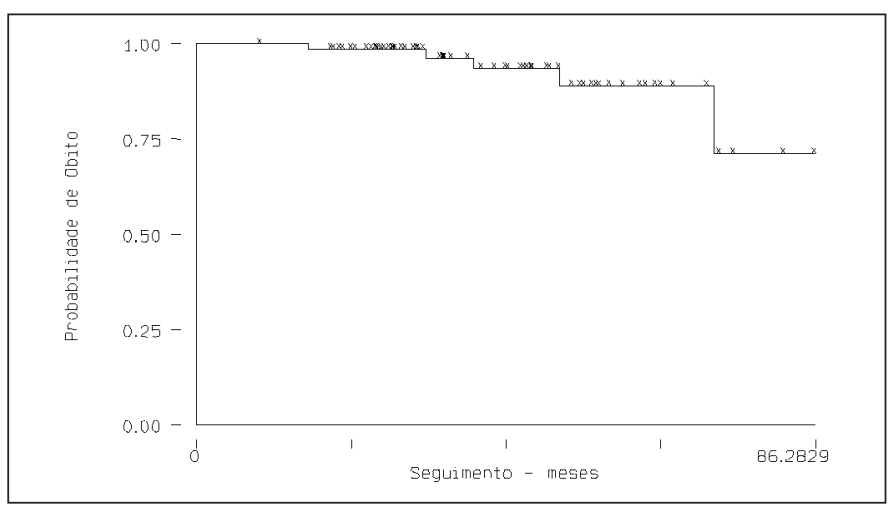

Figura 2 - Probabilidade de óbito. Curva atuarial

Em relação ao tempo de tratamento, observamos que os pacientes tratados por um período $\leq 157$ horas apresentaram maior índice de complicações e menor índice de conservação quando comparados àqueles tratados por um tempo $>157$ horas $(\mathrm{P}=0,047$ e $\mathrm{P}=0,726)$; não existiu significância estatística para a conservação (tabelas 2 e 3 ).

\section{DISCUSSÃO}

Essa amostra brasileira apresentou distribuição quanto ao sexo, idade e raça semelhantes àqueles observados na literatura mundial ${ }^{(2-3)}$.

A preocupação com o montante de retenção da função visual pós-tratamento braquiterápico é evidente desde os primeiros relatos ${ }^{(19)}$, porém a quantidade de acuidade visual considerada, por cada autor, como "preservação de visão útil" é bastante variável e subjetiva. De maneira geral, podemos considerar que resultados visuais satisfatórios foram observados utilizando-se os diferentes isótopos disponíveis, em 30\% a $75 \%$ dos $\operatorname{casos}^{(12,16-18)}$. A deterioração da acuidade visual inicia-se, na maioria dos pacientes, 2 anos após o tratamento, sendo causada principalmente pela presença de complicações 


\begin{tabular}{|c|c|c|c|c|c|}
\hline \multirow{3}{*}{$\begin{array}{l}\text { Variável } \\
\text { Altura (mm) }\end{array}$} & \multicolumn{3}{|c|}{$\begin{array}{l}\text { Variável ( } n \text { total do grupo) } \\
\text { \% conservação ( } n \text { ' conservação do grupo) }\end{array}$} & & \multirow{3}{*}{$\begin{array}{c}\text { Estudo estatístico } \\
\text { Person } \text { Chi' }^{2}(2) \\
\text { Fisher's exact } \\
\begin{array}{c}P=0,007 \\
F=0,005\end{array}\end{array}$} \\
\hline & $\leq 3(15)$ & $>3 \leq 5(17)$ & $>5 \leq 8(27)$ & $>8$ (10) & \\
\hline & $100 \%(15)$ & $76,47 \%(13)$ & $77,78 \%(21)$ & $40 \%(4)$ & \\
\hline \multirow[t]{2}{*}{ Base $(\mathrm{mm})$} & $<10(23)$ & $\geq 10<15$ (42) & $\geq 15$ (4) & - & $P=0,011$ \\
\hline & $91,30 \%(21)$ & $73,81 \% \quad(31)$ & $25 \%(1)$ & - & $F=0,015$ \\
\hline \multirow[t]{2}{*}{ Ápice cGy } & $<12.000(45)$ & $\geq 12.000(24)$ & - & - & $P=0,033$ \\
\hline & $68,89 \%(31)$ & $91,67 \%(22)$ & - & - & $F=0,039$ \\
\hline \multirow[t]{2}{*}{ cGg/hora ápice } & $>45(27)$ & $>45(42)$ & - & - & $P=0,029$ \\
\hline & $62,96 \%(17)$ & $85,71 \%(36)$ & - & - & $F=0,041$ \\
\hline \multirow[t]{2}{*}{ Base cGy } & $\leq 25.000(28)$ & $>25.000 \leq 35.000(27)$ & $>35.000(14)$ & - & $P=0,274$ \\
\hline & $85,71 \%(24)$ & $74,07 \%(20)$ & $64,29 \%(9)$ & - & $F=0,246$ \\
\hline \multirow[t]{2}{*}{ cGy hora/base } & $\leq 120(38)$ & $>120$ (31) & - & - & $P=0,006$ \\
\hline & $89,47 \%(34)$ & $61,29 \%(19)$ & - & - & $F=0,009$ \\
\hline \multirow{2}{*}{$\begin{array}{l}\text { Tempo } \\
\text { tratamento (hs) }\end{array}$} & $\leq 157(16)$ & $>157$ (53) & - & - & $P=0,726$ \\
\hline & $72,73 \%(8)$ & $77,59 \%(45)$ & - & - & $F=0,708$ \\
\hline \multirow{2}{*}{$\begin{array}{l}\text { Atividade placa } \\
(\mathrm{mCi})\end{array}$} & $\leq 2(36)$ & $>2$ (33) & - & - & $P=0,002$ \\
\hline & $91,67 \%$ (33) & $60,61 \%(20)$ & - & - & $F=0,004$ \\
\hline
\end{tabular}

\begin{tabular}{|c|c|c|c|c|c|}
\hline \multirow{3}{*}{$\begin{array}{l}\text { Variável } \\
\text { Altura (mm) }\end{array}$} & \multicolumn{3}{|c|}{$\begin{array}{l}\text { Variável (n total do grupo) } \\
\text { \% complicação (n' complicação do grupo) }\end{array}$} & & \multirow{3}{*}{$\begin{array}{c}\text { Estudo estatístico } \\
\text { Person } \text { Chi }^{2}(2) \\
\text { Fisher's exact } \\
\begin{array}{c}P=0,031 \\
F=0,024\end{array}\end{array}$} \\
\hline & $\leq 3(15)$ & $>3 \leq 5(17)$ & $>5 \leq 8(27)$ & $>8$ (10) & \\
\hline & $53,33 \%(8)$ & $70,59 \%(12)$ & $92,59 \%(25)$ & $80 \%(8)$ & \\
\hline \multirow[t]{2}{*}{ Base $(\mathrm{mm})$} & $<10(23)$ & $\geq 10<15(42)$ & $\geq 15$ (4) & - & $P=0,376$ \\
\hline & $69,57 \%(16)$ & $78,57 \%$ (33) & $100 \%(4)$ & - & $F=0,510$ \\
\hline \multirow[t]{2}{*}{ Ápice cGy } & $<12.000(45)$ & $\geq 12.000$ (24) & - & - & $P=0,008$ \\
\hline & $86,67 \%$ (39) & $58,33 \%(14)$ & - & - & $F=0,015$ \\
\hline \multirow[t]{2}{*}{ cGg/hora ápice } & $\leq 45(27)$ & $>45$ (42) & - & - & $P=0,057$ \\
\hline & $88,89 \%(24)$ & $69,05 \%(29)$ & - & - & $F=0,080$ \\
\hline \multirow[t]{2}{*}{ Base cGy } & $\leq 25.000(28)$ & $>25.000 \leq 35.000$ & $>35.000(14)$ & - & $P=0,309$ \\
\hline & $67,86 \%(19)$ & $85,19 \%(23)$ & $78,57 \%(11)$ & - & $F=0,320$ \\
\hline \multirow[t]{2}{*}{ cGy hora/base } & $\leq 120$ & $>120(31)$ & - & - & $P=0,016$ \\
\hline & $65,79 \%(25)$ & $90,32 \%(28)$ & - & - & $F=0,022$ \\
\hline Tempo & $\leq 157(11)$ & $>157(58)$ & - & - & $P=0,047$ \\
\hline tratamento (hs) & $100 \%(11)$ & $72,41 \%(42)$ & - & - & $F=0,056$ \\
\hline Atividade placa & $\leq 2(36)$ & $>2(33)$ & - & - & $P=0,008$ \\
\hline$(\mathrm{mCi})$ & $63,89 \%(23)$ & $90,91 \%(30)$ & - & - & $F=0,010$ \\
\hline
\end{tabular}

radiogênicas ${ }^{(27)}$. Em nossa amostra o tratamento causou diminuição da acuidade visual em $37,2 \%$ da amostra.

Ocorreu diminuição da altura do tumor em $79,7 \%$ dos nossos casos e do diâmetro basal em $66,7 \%$, o que nos parece dentro da expectativa de resposta tumoral local para o método, sendo que $13 \%$ dos casos, foram considerados planos por ocasião da última revisão. Aparentemente a resolução da massa tumoral para uma cicatriz coroidoretiniana plana ocorre somente no tratamento de lesões pequenas. Enquanto alguns autores $^{(28)}$ observaram que a maioria dos tumores apresenta uma regressão lenta e parcial, com persistência de massa tu- moral residual em $50 \%$ do seu tamanho original ${ }^{(28)}$, outros observaram que a maioria dos tumores com espessura inicial menor que $5 \mathrm{~mm}$ apresentaram regressão tumoral superior a $50 \%$ em 18 meses, enquanto aqueles com mais de $5 \mathrm{~mm}$ tiveram regressão inferior a $50 \%{ }^{(16)}$.

Consideramos em nosso trabalho como melhor resposta local ao tratamento os parâmetros que cursaram com os maiores índices de conservação associados aos menores índices de complicações.

A literatura tem demonstrado que as principais complicações associadas à braquiterapia são as tardias e que sua 
incidência está em torno de $40 \%$ (3,8,10,19,29). Fatores aparentemente envolvidos são: o tamanho e a localização do tumor tratado assim como o isótopo e a dose radioativa utilizada. Acredita-se que estas complicações são menos freqüentes quando utilizamos placas com isótopos radioativos de baixa energia (Ex: Iodo 125, o Rutênio 106 e o Iridium 192), que possibilitam maior direcionamento da radiação para o tecido tumoral $^{(3)}$. A utilização de Iodo 125 demonstrou um baixo índice de complicações (34\%), tendo sido atribuido este resultado às características intrínsecas deste isótopo ${ }^{(29)}$. Outro relato demonstrou significante maior índice de complicações no tratamento de lesões com mais de $8 \mathrm{~mm}$ de altura ${ }^{(24)}$.

Em nossa amostra, o índice total de complicações pode ser considerado alto $(76,8 \%)$, este fato, ao nosso ver, deve-se ao isótopo utilizado (Cobalto 60) e ao tamanho das lesões tratadas.

Com intuito de diminuir o alto índice de complicações, novas associações terapêuticas como a termoradioterapia, que associa a hipertermia à braquiterapia tem demonstrado resultados satisfatórios por permitir a utilização de doses 30 a $50 \%$ menores $^{(30)}$.

$\mathrm{O}$ insucesso no tratamento, devido tanto à falha no controle tumoral local como pela presença de complicações incompatíveis com a manutenção do globo, são indicativas de enucleação; na literatura esta ocorrência está entre $6 \%$ a $20 \%{ }^{(30-31)}$, em nossa amostra a incidência foi de $23,2 \%$.

Nosso índice de falha no controle tumoral local $(14,5 \%)$ foi bastante próximo ao de outros estudos com Cobalto 60: $12 \%$ a $33 \% \%^{(20,22-23)}$. Para o Iodo 125 , os resultados pouco diferem: $14 \%$ e $22 \%{ }^{(15,21)}$.

Os nossos resultados com referência à frequiência de doença metastática $(11,6 \%)$ são semelhantes a alguns relatos $(12 \%$ em 5 anos $)^{(15)}$ e melhores que outros, que apresentaram $18 \%$ em 3,3 anos de seguimento, tendo estas ocorrido em média 24 meses após o tratamento ${ }^{(26)}$. Em nossa amostra, a ocorrência de doença metastática ocorreu em média, 31,8 meses após o tratamento.

Os índices de sobrevida projetados para 5 e 7 anos em nossa amostra, foram de respectivamente, $89,1 \%$ e $71,3 \%$, valores semelhante aos referidos na literatura para o méto$\mathrm{do}^{(6,12,24)}$. Alguns autores encontraram taxas de sobrevida utilizando braquiterapia comparáveis às observadas após enucleação para tumores com tamanhos equivalentes ${ }^{(29)}$.

A variável que apresentou maior influência na determinação da melhor resposta local foi a altura da lesão tratada. As lesões com até $3 \mathrm{~mm}$ de altura apresentaram resposta local significantemente melhores que as demais. Para as lesões de até $5 \mathrm{~mm}$ de altura, os resultados ainda puderam ser considerados bons, entre 5 e $8 \mathrm{~mm}$ relativamente satisfatórios, devido à boa conservação, porém elevado índice de complicações e acima de $8 \mathrm{~mm}$, o método se mostrou pouco eficiente, com índice de conservação baixo e de complicações elevado. Portanto, quanto maior a altura, menor foi a eficácia do método.

A literatura tem demonstrado a importância da altura da lesão no índice final de sucesso do tratamento braquiterápico, sendo os melhores resultados observados no tratamento de tumores com até 5-6mm de espessura ${ }^{(16,24)}$.

Em relação ao diâmetro basal, observamos que a maior efetividade do método ocorreu no tratamento de lesões menores que $10 \mathrm{~mm}$ de base, entre $10 \mathrm{~mm}$ e $15 \mathrm{~mm}$ os resultados foram relativamente satisfatórios, porém acima de $15 \mathrm{~mm}$ os resultados foram pobres devido à baixa conservação e alto índice de complicações.

Poucas amostras na literatura utilizaram o mesmo isótopo por um tempo suficientemente grande para ultrapassar a sua meia vida, permitindo reunir no mesmo grupo, o tratamento de lesões com o mesmo material radioativo, em diversas fases de seu rendimento físico. Esta amostra teve estas características.

Em relação à dose radioativa emitida ao ápice do tumor, foi evidente a melhor resolução do método na utilização de altas doses (>12.000cGy). Alguns relatos demonstram a influência da dose radioativa total absorvida pelo ápice nos resultados do tratamento braquiterápico, tendo sido observado incidência de falha no controle tumoral 8 vezes menor nos pacientes tratados com uma dose no ápice maior que $7.000 \mathrm{cGy}{ }^{(15)}$. Outros autores observaram que regressão completa foi encontrada em $66 \%$ dos tumores tratados com dose maior que 15.000cGy; entretanto, este grupo teve maior índice de complicações $^{(17)}$.

Quanto à irradiação da base, a melhor resposta terapêutica ocorreu com a utilização de baixas doses.

Alguns estudos demonstram interesse na análise dos efeitos da taxa de dose utilizada, para alguns autores essa taxa pode influir na recuperação celular e na progressão do ciclo celular. Taxas de doses baixas e contínuas podem possibilitar o reparo celular ao dano radioterápico, o que é chamado reparo de dano subletal. Os estudos das células do melanoma sugerem maior radiosensibilidade a altas taxas de dose, entretanto, o impacto desta para o tecido normal deve ser levado em conta, pois um aumento na morbidade, traria desvantagens no resultado terapêutico final ${ }^{(31)}$.

Neste estudo a melhor resolução foi observada com taxas de dose apicais maiores que $45 \mathrm{cGy} / \mathrm{h}$, achado este semelhante aos de alguns relatos, cujos melhores resultados ocorreram na utilização de taxa de dose apical maior que $40 \mathrm{cGy} / \mathrm{h}^{(14)}$, enquanto outros autores encontraram incidência de falha do controle local 4,75 vezes maiores na utilização de taxas de dose apicais menores que $50 \mathrm{cGy} / \mathrm{h}^{(15)}$.

Em relação à base, observamos melhor resolução do método com taxas de dose menores que $120 \mathrm{cGy} / \mathrm{h}$. Alguns relatos, entretanto, demonstraram ao utilizar Iodo125, que olhos tratados com taxa de dose na base menor que $200 \mathrm{cGy} / \mathrm{h}$ apresentaram índice de falha do controle local três vezes maior do que o grupo que recebeu taxas superiores ${ }^{(15)}$.

A taxa de dose na base é uma característica física que pode ser manipulada no planejamento braquiterápico de isótopos como o Iodo 125, pois esta depende, apenas, da sua atividade. Para estes isótopos de decaimento rápido (meia vida curta), gerados em sementes, é possível construir uma configuração, para obtenção de determinada taxa de dose em cada aplicador, 
variando o número, a disposição e a atividade das sementes, pois estes aplicadores (placas) são manufaturados individualmente para cada caso. Ter a possibilidade de trabalhar dentro de diferentes faixas de taxas de dose é uma forma de otimização do método. No caso do Cobalto 60, o isótopo é parte integrante do aplicador, não permitindo qualquer manipulação.

As lesões tratadas com placas com atividade menor que 2 $\mathrm{mCi}$ foram as que apresentaram melhores resultados. Comparando-se a utilização de placas de Iodo 125 de baixa e alta atividade, alguns autores observaram que alta atividade cursou com maior índice de controle tumoral e de complicações $^{(32)}$, enquanto outros observaram que os pacientes tratados por placas menos ativas apresentaram melhor prognóstico ${ }^{(33)}$.

A duração do tratamento depende do tamanho da lesão e da atividade da placa. Quanto maior a lesão ou menor a atividade da placa, maior o tempo de tratamento. Nossos melhores resultados foram observados num tempo de tratamento maior que 157 horas; outros autores, entretanto, observaram maior índice de falha de controle tumoral quanto maior foi o tempo de tratamento (154 horas) ${ }^{(15)}$. Talvez este fato deva-se aos diferentes isótopos utilizados, já que para os isótopos com meia vida curta, onde existe uma constante renovação do material radioativo, o tempo de tratamento é reduzido e homogêneo quando comparado ao Cobalto 60; este tempo foi muito variável em nossa amostra (116 a 481h).

Acreditamos que futuras investigações devem dar maior atenção ao impacto que as diferentes características próprias do método radioterápico como doses e taxas de doses possam ter nos resultados, para que possam ser incluídos no processo de planejamento.

Aparentemente, múltiplos fatores influem no resultado do tratamento braquiterápico. A otimização do método é amplamente discutida na atualidade. $\mathrm{O}$ conhecimento dos melhores parâmetros utilizados no tratamento dos nossos pacientes, com vistas à otimização dentro das nossas condições de trabalho e isótopos disponíveis, motivaram este trabalho.

\section{CONCLUSÃO}

Os melhores resultados foram observados no tratamento de melanomas menores que $5 \mathrm{~mm}$ de altura e $10 \mathrm{~mm}$ de diâmetro basal, com a utilização de dose no ápice maior que $12.000 \mathrm{cGy}$ e taxa de dose no ápice maior que $45 \mathrm{cGy} / \mathrm{hora}$, dose na base menor que 25.000cGy e taxa de dose na base menor que $120 \mathrm{cGy}$, tempo de tratamento menor que 157 horas e atividade da placa menor que $2 \mathrm{mCi}$.

\section{ABSTRACT}

Purpose: To determine prognostic factors for local control of uveal melanoma treated by brachytherapy with cobalt 60 . Methods: We evaluated indexes of local tumor control, complications, metastatic disease and global survival in 69 uveal melanoma patients treated with brachytherapy with cobalt 60 from November 1988 to October 1994, at the Hospital do Câncer - A. C. Camargo, São Paulo. We confronted these indexes with different tumoral characteristics and brachytherapy techniques for the determination of the best prognostic factors. Results: After treatment decrease in height was observed in $55(79.9 \%)$ lesions, in basal diameter in $46(66.7 \%)$, and in visual acuity in $26(37.2 \%)$. $16(23.2 \%)$ eyes were later enucleated due to failure of tumor control in $10(62.5 \%)$ or complications in $6(37.5 \%)$; enucleations were performed on an average 21.1 months after treatment. The mean follow-up time was 44.3 months (10.1-114.3 months). Metastatic disease occurred in $8(11.6 \%)$ patients and the overall five year survival was $89.1 \%$. Complications occurred in $53(76.8 \%)$ patients and eyeball conservation was accomplished in 53 (76.8\%). Prognostic factors associated with eyeball conservation and lower complication indexes were: lesion thickness less than $5 \mathrm{~mm}$; basal diameter less than $10 \mathrm{~mm}$; apex doses higher than 12,000 cGy; apex dose tax higher than $45 \mathrm{cGy} / \mathrm{h}$; basal dose lower than 25,000 cGy; basal dose rate lower than $120 \mathrm{cGy} / \mathrm{h}$; and plaque activity lower than $2 \mathrm{mCi}$. Conclusion: Tumor size, plaque activity, basal and apex doses and dose rate should be carefully observed in brachytherapy for uveal melanomas in other to optimize its results.

Keywords: Melanoma/drug therapy; Uveal neoplasms/drug therapy; Cobalt/therapeutic use; Prognosis

\section{REFERÊNCIAS}

1. Graham BJ, Duane TD. Ocular melanoma task force report. Am J Ophthalmol 1980;90:728-33.

2. Seddon JM, Egan KM, Gragoudas ES et al. Epidemiology of uveal melanoma. In: Ryan SJ editors. Retina. St. Louis: C. V. Mosby; 1989. p.639-46.

3. Shields JA, Shields CL. Intraocular tumors: A text and atlas. Philadelphia: Saunders; 1992.

4. Osório LA. Estudo clínico dos tumores da úvea. An Oftalmol 1984;3:31-8.

5. Char DH. Metastatic choroidal melanoma. Am J Ophthalmol 1978;86:76-80.

6. Augesburger JJ, Gamel JW, Lauritzen K, Brady LW. Cobalt-60 plaque radioterapy versus enucleation for posterior uveal melanoma. Am J Ophthalmol 1990;109:585-92.

7. Shields JA, Shields CL, Donoso LA - Management of posterior uveal melanoma - Major review. Surv Ophthalmol 1991;36:161-95.

8. Brady LW, Shields JA, Augesburger J, Markoe A, Karlsson UL, Complications from radiation therapy to the eye. Front Radiat Ther Oncol 1989;23:238-50.

9. Anderson LL, Tsao STC. Physical aspects of eye plaque brachytherapy using photon emitters. In: Alberti WE, Sagerman RH. Radiotherapy of intraocular and orbital tumors. Heidelberg: Springer-Verlag; 1993. p.347-61.

10. Lommatzsch PK. Results after B-irradiation (106-Ru/106-Rh) of choroidal melanomas: 20 year's experience. Br J Ophthalmol 1986;70:844-51.

11. Hall EJ, Brenner DJ. The dose-rate effect revisited: radiobiological consideration of importance in radiotherapy. Int J Radiat Oncol Biol Phys 1991;21:1403-14.

12. Valcárcel F, Valverde S, Cárdenes H, Cajigal C, De La Torre A, Magallón R et al. Episcleral iridium-192 wire therapy for choroidal melanomas. Int J Radiat Oncol Biol Phys 1994;30:1091-7.

13. Earle J, Kline RW, Robertson DM. Selection of iodine-125 for the collaborative ocular melanoma study. Arch Ophthalmol 1987;105:763-4.

14. Rotman M, Long RS, Packer S, Moroson H, Galin MA, Chan B. Radiation therapy of choroidal melanoma. Trans Ophthalmol Soc UK 1977;97:431-5. 
15. Quivey J, Augsburger J, Snelling L, Brady LW. 125 I plaque therapy for uveal melanoma: analisys of the impact of time and dose factors on local control. Cancer 1996;77:2356-62.

16. Grange JD, Gerard JP, Ragab M, Quintero P, Delaroche G, Jean-Louis B, et al. Evalution of 100 melanomas of the choroid and ciliary body treated by apllication of ruthénium (RU106/RH106). - Bul Soc Ophthalmol Fr 1989;89:679-82

17. Tijho-Heslinga RE, Kakebeeke-Kemme HM, Davelaar J, De Vromme H, Bleeker JC, Oosterhuis JA, Leer JW. Results of ruthénium irradiation of uveal melanomas. Radiother Oncol 1993;29:33-8.

18. Pallazzi MA, Abramson DH. Placas radioativas episclerais em melanoma de coróide. Parte 1: Contribuição ao estudo dos efeitos locais. Arq Bras Oftalmol 1994;57:89-93.

19. Sttallard HB. Radiotherapy for malignant melanoma of the choroid. $\mathrm{Br} \mathrm{J}$ Ophthalmol 1966;50:147-55

20. Char DH. Current treatments and trials in uveal melanoma. Oncology, 1989:3:113-21.

21. Bosworth J, Packer S, Rotman M, Ho T, Finger PT. Choroidal melanoma: 125-I plaque therapy. Radiology 1988;169:249-51.

22. Karlsson U, Augsburger JJ, Shields JA, Markoe AM, Brady LW, Woodleigh R. Recurrence of posterior uveal melanoma after CO-60 episcleral plaque therapy. Opthalmology 1989;96:382-7.

23. Beitler JJ, McCormick B, Ellsworth RM, Abramson DH, Anderson LL, Loffredo C. Ocular melanomas: total dose and dose rate effects with Co-60 plaque therapy. Radiology 1990;176:275-8.

24. Giblin ME, Shields JA, Augsburger JJ, Brady LW. Episcleral plaque radiotherapy for uveal melanoma. Aust N Z J Ophthalmol 1989;17:153-6.
25. Quivey J, Mandell R, Char D, Kroll S, Weaver K. Uveal melanoma: analysis of local treatment failures following $125 \mathrm{I}$ high intensite episcleral plaque therapy. Proc Am Endocuriether Soc 1993;5:40.

26. Summanen P, Immonen I, Heikkonen J, Tommila P, Laatikainen L, Tarkkanen A. Survival of patients and metastatic and local recurrent tumor growth in malignant melanoma of the uvea after ruthénium plaque radiotherapy. Ophthalmic Surg 1993;24:82-90.

27. Kreissig I. Use of the scleral applicator for I 125 in the treatment of choroidal melanoma (long-term results). Cesk Oftalmol 1994;50:135-44.

28. Cruess AF, Augsburger JJ, Shields JA, Brady LW, Markoe AM, Day JL. Regression of posterior uveal melanoma following cobalt 60 plaque radiotherapy. Ophthalmology 1984;91:1716-9.

29. Packer S, Rotman M, Salanitro P. Iodine 125 irradiation of choroidal melanoma. Clinical experience. Ophthalmology 1984;91:1700-8.

30. Petrovich Z, Astrahan M, Luxton G, Shields JA, Shields CL, Brady LW. Primary malignant melanoma of the uvea: Radioactive plaque therapy and other treatment modalities. In: Alberti WE, Segerman RH. Radiotherapy of intraocular and orbital tumors. Heidelberg: Springer-Verlag; 1993. p.31-44.

31. Shields CL, Shields JA, Karlsson U, Markoe AM, Brady LW. Reasons for enucleation after plaque radiotherapy for posterior uveal melanoma. Clinical findings. Ophthalmology 1989;96:919-23.

32. Yang CM, Olsen KR, Schwade JG, Houdek PV, Markoe AM, Pisciotta V et al. Dose rate effect of 125-I irradiation on normal rabbit eyes and experimental choroidal melanoma. Exp Eye Res 1993;57:577-85.

33. Kleineidan M, Guthoff R. Possible effects of radiobiological parameters on metastatic spread of uveal melanomas treated with 106RU plaques. Ger J Ophthalmol 1994;3:22-5.

\title{
ABO ELETRÔNICO
}

\section{A versão eletrônica dos Arquivos Brasileiros de} Oftalmologia com textos completos está disponivel em:

\author{
- $\mathrm{ABO}$ - Arquivos Brasileiros de Oftalmologia \\ http://www.abonet.com.br
}

- SciELO - Scientific Electronic Library Online http://www.scielo.org

- Free Medical Journals - http://www.freemedicaljournals.com 